\title{
In vivo histotripsy brain treatment
}

\author{
Jonathan R. Sukovich, PhD, ${ }^{1}$ Charles A. Cain, $\mathrm{PhD},{ }^{1}$ Aditya S. Pandey, MD, ${ }^{2}$ \\ Neeraj Chaudhary, MD, ${ }^{3}$ Sandra Camelo-Piragua, MD, ${ }^{4}$ Steven P. Allen, PhD, ${ }^{1}$ \\ Timothy L. Hall, PhD, ${ }^{1}$ John Snell, PhD, ${ }^{5,6}$ Zhiyuan Xu, MD, ${ }^{6}$ Jonathan M. Cannata, PhD, ${ }^{7}$ \\ Dejan Teofilovic, PhD, James A. Bertolina, PhD, ${ }^{7}$ Neal Kassell, MD, ${ }^{5}$ and $\mathrm{Zhen} \mathrm{Xu}, \mathrm{PhD}{ }^{1}$
}

Departments of ${ }^{1}$ Biomedical Engineering, ${ }^{2}$ Neurosurgery, ${ }^{3}$ Radiology, and ${ }^{4}$ Pathology, University of Michigan, Ann Arbor, Michigan; ${ }^{5}$ Focused Ultrasound Foundation, Charlottesville, Virginia; ${ }^{6}$ University of Virginia, Department of Neurosurgery, Charlottesville, Virginia; and ${ }^{7}$ HistoSonics, Inc., Ann Arbor, Michigan

\begin{abstract}
OBJECTIVE Histotripsy is an ultrasound-based treatment modality relying on the generation of targeted cavitation bubble clouds, which mechanically fractionate tissue. The purpose of the current study was to investigate the in vivo feasibility, including dosage requirements and safety, of generating well-confined destructive lesions within the porcine brain utilizing histotripsy technology.
\end{abstract}

METHODS Following a craniectomy to open an acoustic window to the brain, histotripsy pulses were delivered to generate lesions in the porcine cortex. Large lesions with a major dimension of up to $1 \mathrm{~cm}$ were generated to demonstrate the efficacy of histotripsy lesioning in the brain. Gyrus-confined lesions were generated at different applied dosages and under ultrasound imaging guidance to ensure that they were accurately targeted and contained within individual gyri. Clinical evaluation as well as MRI and histological outcomes were assessed in the acute ( $\leq 6$ hours) and subacute ( $\leq 72$ hours) phases of recovery.

RESULTS Histotripsy was able to generate lesions with a major dimension of up to $1 \mathrm{~cm}$ in the cortex. Histotripsy lesions were seen to be well demarcated with sharp boundaries between treated and untreated tissues, with histological evidence of injuries extending $\leq 200 \mu \mathrm{m}$ from their boundaries in all cases. In animals with lesions confined to the gyrus, no major hemorrhage or other complications resulting from treatment were observed. At 72 hours, MRI revealed minimal to no edema and no radiographic evidence of inflammatory changes in the perilesional area. Histological evaluation revealed the histotripsy lesions to be similar to subacute infarcts.

CONCLUSIONS Histotripsy can be used to generate sharply defined lesions of arbitrary shapes and sizes in the swine cortex. Lesions confined to within the gyri did not lead to significant hemorrhage or edema responses at the treatment site in the acute or subacute time intervals.

https://thejns.org/doi/abs/10.3171/2018.4.JNS172652

KEYWORDS focused ultrasound; histotripsy; thrombolysis; intracerebral hemorrhage; neurosurgery

$\mathrm{N}$ ONINVASIVE surgical techniques are favored in the treatment of brain pathologies as they do not require cerebral exposure and retraction, and avoid complications related to invasive procedures. Numerous technologies have been investigated ${ }^{1,10,11,20,35,44}$ to address the various cerebral surgical needs, including tumor ablation, ${ }^{5,23}$ lesioning for movement disorders, ${ }^{7}$ and hematoma evacuation following hemorrhagic stroke. ${ }^{25,26}$ MRI-guided high-intensity focused ultrasound (HIFU) thermal therapies $^{3,14-17}$ have recently shown success in the treatment of essential tremor ${ }^{7}$ and chronic neuropathic pain. ${ }^{18}$ However, HIFU thermal therapies are limited in the range and scope of targets due to dissipation of ultrasound energy and heating of the skull when treating large or superficial lesions. 2,6,9,24,29-32,41

In this study, we present a novel, nonthermal ultrasound therapy called intrinsic threshold histotripsy (hereafter referred to only as "histotripsy") for use as a lesioning therapy in brain applications. Histotripsy is an ultrasound therapy that uses short-duration, high-amplitude ultrasound

ABBREVIATIONS H \& E = hematoxylin and eosin; HIFU = high-intensity focused ultrasound; ICH = intracerebral hemorrhage.

SUBMITTED October 23, 2017. ACCEPTED April 23, 2018.

INCLUDE WHEN CITING Published online October 12, 2018; DOI: 10.3171/2018.4.JNS172652. 
pulses ( $\geq 30 \mathrm{MPa}$ ), delivered using an extracorporeal ultrasound transducer, to generate targeted bubble clouds within tissues. $22,27,43$ These bubble clouds are generated via the deposition of ultrasound energy alone, without requiring the introduction of external nucleation agents such as injected microbubbles. Damage during histotripsy is produced by the rapid expansion and collapse of the generated bubbles. This process causes very large, highly localized mechanical stresses and strains in the targeted tissues, which act to mechanically fractionate and liquefy them. Whereas HIFU thermal therapies rely on thermal necrosis to destroy tissues while preserving their structure, the mechanical damage generated during histotripsy destroys tissues at a cellular level, reducing them to a liquefied homogenate of acellular debris. The short duration of the histotripsy pulses ( $\leq 2$ acoustic cycles), which limits their physical width, severely restricts the spatial extent over which pressures sufficient for bubble cloud generation may be reached, resulting in small focal volumes and allowing highly targeted lesions to be generated during histotripsy treatment without damaging surrounding tissues. Indeed, histotripsy has been demonstrated to generate precisely defined lesions with sharp boundaries between treated and untreated regions ${ }^{27,42,43}$ in a wide range of tissues, ${ }^{37,38,40,45,46}$ and clinical trials are ongoing utilizing histotripsy to treat liver disorders.

The narrow width of the histotripsy pulses used during therapy also highly restricts the degree to which induced aberrations, even in highly aberrating media such as the skull, ${ }^{9}$ can distort and shift the therapy focus, as aberrations large enough to result in focal shifts greater than the width of the ultrasound pulses preferentially prevent cavitation and damage generation instead. Indeed, histotripsy has been demonstrated to generate targeted, well-confined lesions with millimeter accuracy through the skull ${ }^{19}$ even without aberration correction. ${ }^{33}$ The short duration of the pulses also allows histotripsy to be delivered rapidly while maintaining low duty cycles $(<<0.01 \%)$, which limits heating in tissues in the ultrasound beam path. This can allow histotripsy therapy to be delivered much more quickly ${ }^{12}-$ and to more superficial targets ${ }^{34}$ without unsafe tissue heating - than is possible with HIFU thermal therapies, as well as to targets through skulls whose conformations would otherwise prevent sufficient amounts of HIFU thermal energy from being deposited at the focal target before generating unsafe heating elsewhere. These features have the potential to allow histotripsy to be applied utilizing preoperative MRI or CT studies ${ }^{21,28}$ combined with ultrasound feedback for treatment targeting and guidance as opposed to real-time MR thermometry; this would impose less restrictive conditions on its use. The aim of this study was to utilize a modified porcine animal model ${ }^{8}$ to determine the safety and efficacy of generating targeted lesions within the brain using histotripsy. In particular, it was designed to determine perilesional edema, hemorrhage, and mechanical injury after lesion formation.

\section{Methods}

This study was approved by the Institutional Care and Animal Use Committee at the University of Virginia and the University Committee on Use and Care of Animals at the University of Michigan. Procedures were performed at 2 locations, the University of Michigan animal surgery and operating rooms and the University of Virginia animal surgery suite. Histotripsy treatments were delivered to 2 cohorts of adolescent pigs in this study to monitor the acute ( $\leq 6$ hours) and subacute (72 hours) effects of histotripsy applied to the brain. The acute and subacute cohorts consisted of 10 and 4 animals, respectively, and all animals used during these studies weighed approximately 15 $\mathrm{kg}$. All acute procedures were performed at the University of Michigan. All subacute procedures were performed at the University of Virginia to take advantage of the group's previous experience and expertise using the swine model for HIFU brain therapies ${ }^{6}$ and their facilities for the procedures.

\section{Histotripsy Treatments}

Histotripsy therapy was delivered through a craniectomy using a custom-built 6-element histotripsy transducer with a center frequency of $1.5 \mathrm{MHz}$ (Fig. 1 left) and a focal spot size of $1.5 \times 1.5 \times 2.5 \mathrm{~mm}$. All treatments were delivered to the cortex of the brain at depths of between 5 and $20 \mathrm{~mm}$ from the exposed surface using histotripsy pulses with estimated ${ }^{21}$ peak rarefactional pressures of 45 $\mathrm{MPa}$. The histotripsy pulses used to generate lesions were delivered at a fixed rate of $10 \mathrm{~Hz}$ in all cases.

Prior to treatment, pigs were sedated with an intramuscular injection of tiletamine (Telazol, $6 \mathrm{mg} / \mathrm{kg}$ ) mixed with xylazine $(2.2 \mathrm{mg} / \mathrm{kg})$, followed by tracheal intubation and inhalation of isoflurane gas $(2 \%-3 \%)$. Vital signs were monitored throughout all procedures performed. After anesthetization, the scalp was reflected caudally following the placement of a U-shaped incision, and a $5-\mathrm{cm}$ craniectomy was performed to create a propagation path to the brain for the histotripsy ultrasound pulses. A sterile coupling bath for the histotripsy transducer and ultrasound imaging probe was mounted over the opening in the cranium and filled with degassed sterile saline as the ultrasound coupling medium.

The histotripsy transducer (Fig. 1 left), with a centermounted ultrasound imaging probe (SonixTouch PA74/12 probe, Ultrasonix), was then affixed to a custom 3 -axis positioner and placed within the coupling bath for treatment. Planning images were acquired prior to treatment by scanning the imaging probe over the brain using the positioner. After identifying a target, the transducer's focus was positioned at the target location and treatment was delivered under ultrasound guidance. ${ }^{13,36,39}$ A schematic drawing of the experimental setup is shown in Fig. 1 right.

Following treatment and evaluation, pigs were euthanized with intravenously administered pentobarbital (140$160 \mathrm{mg} / \mathrm{kg}$ ).

\section{Acute Cohort Treatments}

Single lesions were generated within individual gyri to evaluate the feasibility of using histotripsy to generate precise lesions in the brain, to assess the hemorrhagic risk associated with generating well-confined histotripsy lesions, and to evaluate damage as a function of applied his- 

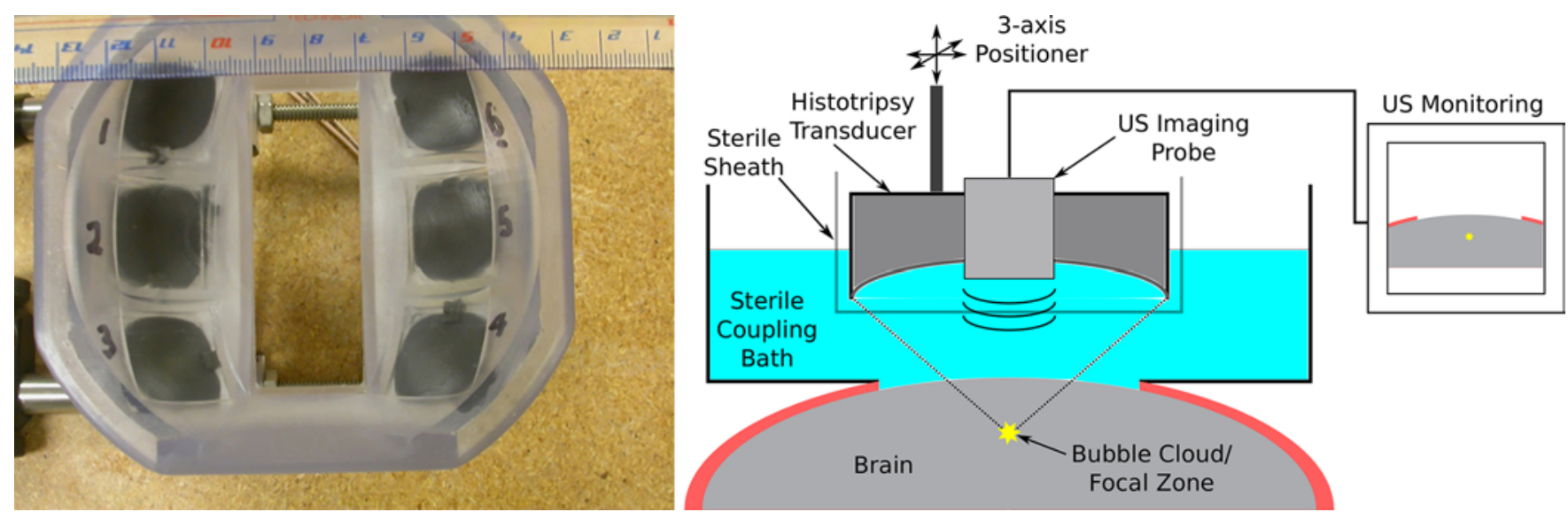

FIG. 1. Left: Photograph of the histotripsy transducer used to deliver treatment in these studies. The ultrasound imaging probe used during treatments for guidance and monitoring was inserted into the central slot visible in this image. Right: Schematic drawing of the setup used during treatment. US = ultrasound. Figure is available in color online only.

totripsy dose, i.e., the number of pulses delivered. Lesions in this study were targeted centrally within the gyri using dosages of 1, 10, 50, and 200 pulses, with corresponding lesioning times of $0.1,1,5$, and 20 seconds.

Lesions with major dimensions of up to $10 \mathrm{~mm}$ were also generated to demonstrate the steerability of histotripsy lesioning, the ability to generate large contiguous lesions, and in order to monitor for potential effects of lesion size on treatment outcomes. Large lesions were generated using the 3-axis positioner to incrementally steer the therapy focus to multiple adjacent and overlapping focal sites within the treatment volume. Spacings between adjacent focal sites of $0.5 \mathrm{~mm}$ and $1.0 \mathrm{~mm}$ were used to assess the degree of overlap required to fully ablate larger volumes. Total lesioning times were $\leq 3$ minutes and $\leq 12$ minutes for all lesions at the $1.0 \mathrm{~mm}$ and $0.5 \mathrm{~mm}$ spacings, respectively, using dosages of $\leq 50$ pulses. An M-shaped lesion was also generated to demonstrate precision lesioning over arbitrary volumes.

Following treatments, pigs were alive for 2 to 6 hours, and follow-up ultrasound images of the lesions were captured to monitor lesioning outcomes. Following euthanasia, the head was removed and T2-weighted fast spin echo MR images of the brain were acquired using a 7-T, small bore, MRI scanner (Agilent Technologies) at the University of Michigan. Following MRI scans, a 3-cm cube of brain tissue containing the treated volume was resected from the skull and placed in 10\% buffered formalin for 3 weeks. In cases where the pig skull was not within the dimensions of the small scanner bore, the $3-\mathrm{cm}$ cube of brain tissue was excised from the skull prior to scanning, immediately after euthanasia, which resulted in an upwelling of blood into the cranium as the brain was being resected. Following MRI and fixing, treated brains were evaluated by gross examination and stained with hematoxylin and eosin ( $\mathrm{H} \& \mathrm{E})$ for histological analysis by a pathologist.

\section{Subacute Cohort Treatments}

Two lesions were generated in the brains of each of the 4 pigs in the subacute cohort. All lesions were targeted centrally within the first and second gyri from the mid- line, with 1 lesion per gyrus. In pigs 1 and 2, single focalsite lesions were generated using applied dosages of 10 and 50 pulses. In pigs 3 and 4, one single focal-site lesion and one large lesion were generated using dosages of 50 pulses per site within each lesion. The large lesions were generated to measure $2.5 \times 2.5 \times 3.5 \mathrm{~mm}$ (pig 3 ) and $2.5 \times$ $3.5 \times 3.5 \mathrm{~mm}$ (pig 4), with corresponding lesioning times of 3 and 5 minutes.

Following treatment, the scalp was sutured closed and the animal was transported to the focused ultrasound suite for MRI. All MR images of the brains were acquired using the 3-T MRI system (Discovery 750T, GE Healthcare) at the University of Virginia. The following MRI scans were acquired for each animal at 1 hour and 72 hours after treatment: T1-weighted, T2-weighted, T2*-weighted, T2-cube, FLAIR, and gadolinium-enhanced T1-weighted. The animals were transported to a recovery area and monitored by trained veterinary staff for 72 hours between MRI scans.

Following euthanasia, the animals were exsanguinated and the brains were excised whole and fixed in $10 \%$ buffered formalin for 8 weeks. The fixed brains were evaluated by gross examination and stained with $\mathrm{H} \& \mathrm{E}$ for histological analysis by a pathologist.

\section{Results}

Histotripsy lesion formation was successful in all animals, and clear demarcations between treated areas and surrounding tissues were observed. Using ultrasound guidance, we were able to precisely generate single lesions with dimensions as small as $1 \times 1 \times 2 \mathrm{~mm}$ within individual gyri while avoiding creating perforations in the pia mater or sulci. Dosage studies revealed that observable damage was generated by as little as 1 histotripsy pulse, and that 50 pulses were necessary for complete ablation. We were able to generate contiguous lesions spanning multiple gyri with characteristic dimensions on the order of $1 \mathrm{~cm}$ by mechanically repositioning the histotripsy focus. Spacings between adjacent foci of $0.5 \mathrm{~mm}$ were required to fully ablate tissues within larger-volume targets. Both the acute and subacute studies indicated minimal hemor- 


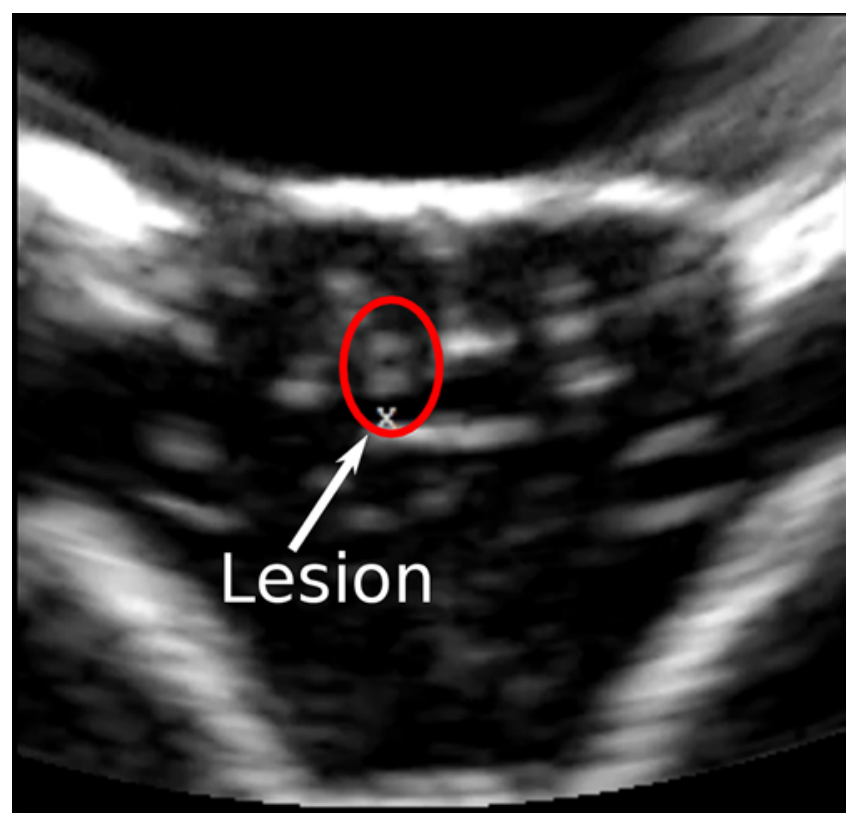

FIG. 2. A typical ultrasound image of the brain following histotripsy treatment. The " $x$ " below the lesion in the center of the image marks the distal end of the histotripsy focal volume and was used to target and guide lesion generation during therapy. The 2 untreated gyri to the right of the lesion, and the one to the left, appear as dark and hypo-echoic regions in ultrasonographic imaging while sulci and midline surrounding it appear bright and hyper-echoic. Following treatment, the lesion is observed as a bright, hyper-echoic region above the focal marker used to guide treatment. Figure is available in color online only.

rhage beyond the lesional area. Edema and inflammatory responses around the generated lesions were observed to be minimal at 72 hours after treatment.

\section{Ultrasound Monitoring of Histotripsy Treatment}

Ultrasound imaging was successfully used to monitor and guide treatments. A typical ultrasound image of the

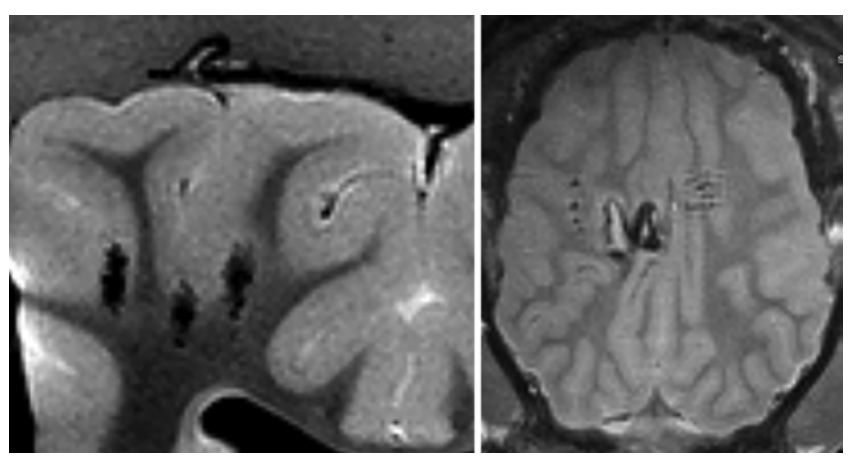

FIG. 3. Typical T2-weighted MR images of lesions generated in the brain following treatment with histotripsy. The brain shown in the image on the left was removed from the skull immediately following euthanasia, prior to decapitation. The brain shown in the image on the right was imaged in situ in the skull. Left: A coronal plane image of 3 single lesions generated by applying 10 histotripsy pulses to each target location in the left half of the brain above the ventricles. Right: An axial plane image of a block M-shaped lesion generated by steering the transducer to create the patterned lesion.
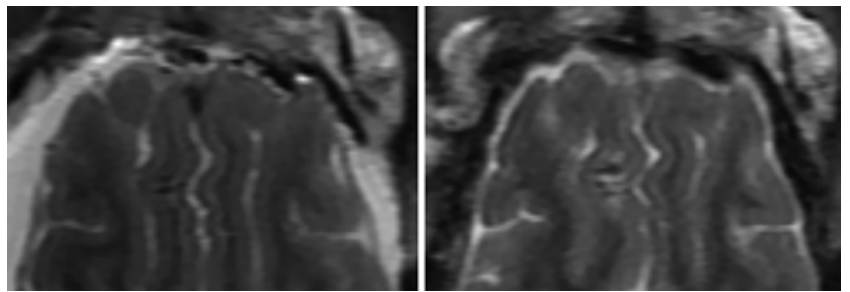

FIG. 4. T2-weighted fast recovery fast spin echo (FRFSE) MR images of pig 4 of the subacute cohort obtained immediately following treatment (left) and at 72 hours after treatment (right). The large lesion is apparent in the cortex in the left hemisphere, the small lesion in the right hemisphere, below the large lesion.

brain following histotripsy lesioning in the gyrus is seen in Fig. 2. In untreated regions (Fig. 2, right side of image), structures including the sulci and midline appear bright and hyper-echoic, while gyri appear hypo-echoic and dark. During treatment, the bubble clouds appear as intensely hyper-echoic regions with time-varying brightnesses, which produce a localized flickering effect where they are generated, allowing easy observation and real-time monitoring and guidance of treatment. Following treatment, lesions (Fig. 2, left side of image) were observed to remain hyper-echoic and visible in ultrasound images for at least 6 hours after treatment, long after any therapy-generated bubbles would have dissolved.

\section{MRI Evaluation of Histotripsy Lesions}

T2-weighted images of the brain revealed hypointense lesions with minimal surrounding damage in both the acute (Fig. 3) and subacute (Fig. 4) cohorts. Edema was observed to be minimal, extending to a maximum of $3 \mathrm{~mm}$ around the large lesion in pig 4 at 72 hours after treatment (Fig. 5), and remaining below $500 \mu \mathrm{m}$ around all other lesions observed in this study. Lesions were seen to be well demarcated and confined (Figs. 3 and 4), even at small separation distances such as between the legs of the M-shaped lesion (Fig. 3 right). The size and delivered dose of the lesions did not affect confinement of damage within the lesional vol-

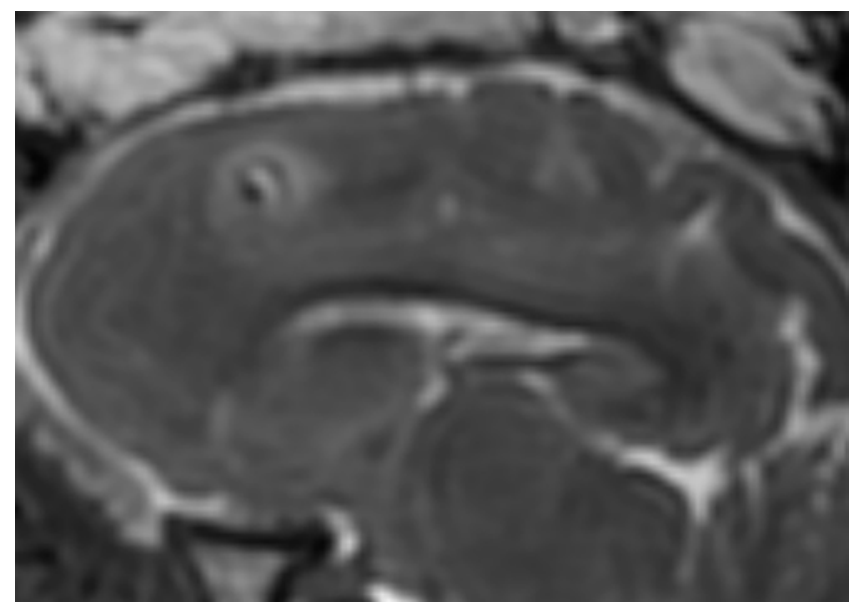

FIG. 5. Sagittal plane T2-cube image of the large lesion in pig 4 of the subacute cohort at 72 hours after treatment showing the presence of edema. 


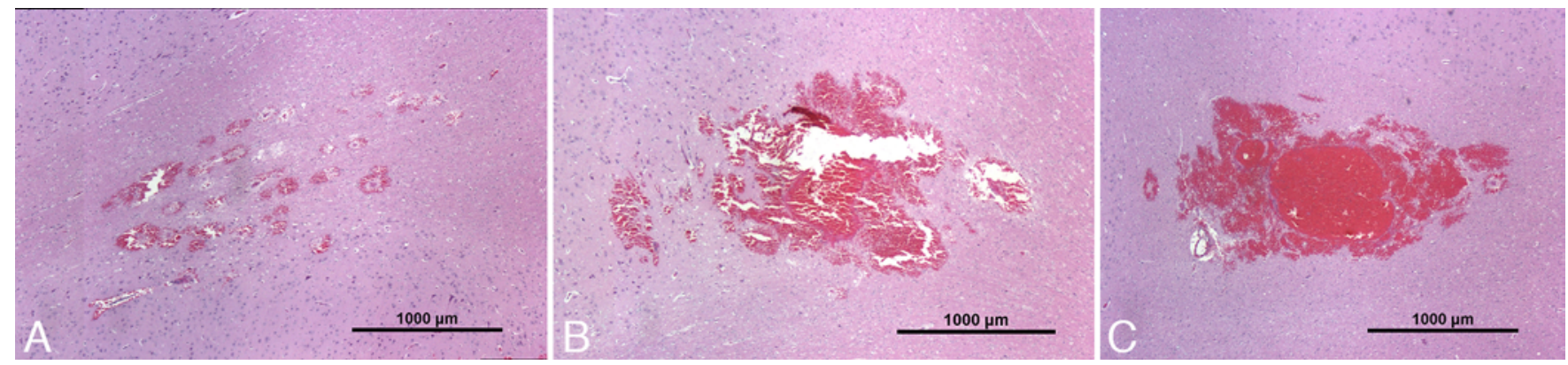

FIG. 6. Photomicrographs of typical H \& E-stained sections showing lesions generated using 1 (A), 10 (B), and 50 (C) histotripsy pulses. Note that the white spaces evident at the centers of the lesions are voids created as homogenized tissue fell out of the tissue during slicing. Figure is available in color online only.

umes. Contrast in all MRI sequences was seen to increase with dose up to an applied dose of 50 pulses. T2 contrast was seen to vary within the volumes of individual large lesions (e.g., the left leg of the M-shaped lesion, Fig. 3 right). This is likely due to the overlapping nature of focal points within the lesions and the infiltration of red blood cells into them as treatment was ongoing, which resulted in lysis of the infiltrated red blood cells via histotripsy and altered their T2 contrast. ${ }^{12} \mathrm{~T} 2 *$ images indicated that hemorrhage remained well confined to within the boundaries of the lesions. T1-weighted images were indicative of localized tissue disruption and confined acute hemorrhage at the lesion sites. Gadolinium-enhanced T1-weighted images showed minimal brightening in the perilesional area, suggesting minimal disruption to the vasculature and blood-brain barrier beyond the lesion.

At 72 hours after treatment, the generated lesions remained apparent on MRI. The single focal-site lesions showed significant contrast reduction on the 72-hour images in comparison to images acquired immediately following treatment in all MR sequences, with the 10-pulse lesions becoming nearly indistinguishable from the surrounding healthy brain tissue. The large lesions generated in pigs 3 and 4 (Figs. 4 right and 5) showed T2 brightening at their boundaries at 72 hours, indicating the presence of edema at the lesion sites. T2* images obtained at 72 hours showed little change compared to initial images and revealed no additional hemorrhage beyond the confines of the generated lesions in all cases. T1 images of the lesions revealed brightening at their peripheries, suggesting the presence of subacute blood. Gadolinium-enhanced T1 images of the large lesions in pigs 3 and 4 showed minor brightening extending $\leq 1 \mathrm{~mm}$ from their boundaries compared to initial images. No such appreciable brightening was observed around the single focal-site lesions.

\section{Histological Evaluation and Dosage Study}

Lesions were composed primarily of hemorrhage and macrophages, with sharply defined boundaries of tissue disruption between treated and untreated tissues. Destruction of cells within the treatment volumes was apparent at all histotripsy doses delivered. The maximum spatial extent of the lesions was observed to remain constant as a function of dose and agreed with the focal dimensions of the transducer $(1.5 \times 1.5 \times 2.5 \mathrm{~mm})$. The degree of damage within the focal volumes was observed to increase with dose, with 1 pulse resulting in damage to approximately $10 \%$ of the tissue within the focal volume (Fig. 6A), 10 pulses resulting in damage to $60 \%$ (Fig. 6B), and 50+ pulses resulting in damage to $\geq 90 \%$ (Fig. 6C). Axonal disruption and ischemic injuries were seen at the boundaries of the lesions, with ischemic injuries extending $\leq 200 \mu \mathrm{m}$ from the lesions' borders. Mild subarachnoid hemorrhage was observed near lesions generated close to boundaries, but it remained well confined only to the area of treatment. Tissues adjacent to lesions were generally unremarkable, with no evidence of secondary effects related to treatment. There were no significant differences between the composition, sharpness of boundaries, or generation of perilesional damage between the single focal-site lesions and large lesions.

Confined hemorrhage into sulci was observed near large lesions that straddled them. However, it is strongly believed that this was significantly affected by the upwelling of blood into the cranium noted during the resection process, as no such significant bleeding was observed in sulci near lesions targeted to avoid them or in brains that were resected after decapitation/exsanguination. A controlled study of the hemorrhagic risk associated with generating lesions over sulci was not within the scope of this initial feasibility study, and future studies are planned to more fully address this risk.

At 72 hours after treatment the lesions remained well demarcated (Figs. 7 and 8) with no evidence of hemorrhage, ischemic changes, or encephalitis beyond the lesional areas. There was no indication of acute inflammation or infection at the lesion sites, and neurons surrounding the lesions were observed to be very viable. Abundant macrophages with bubbly cytoplasm were present in the lesions, indicating active resorption and cleanup processes, and signs of early neovascularization were observed in the cortex lesions. Mild edema was observed around the large lesions in pigs 3 and 4, but the edema did not cause gyral swelling or shifting or other significant anatomical changes. Overall, the histological changes associated with histotripsy-generated lesions resemble those of a confined subacute infarct.

\section{Clinical Assessment: Subacute Cohort}

During the recovery period of the subacute group, 


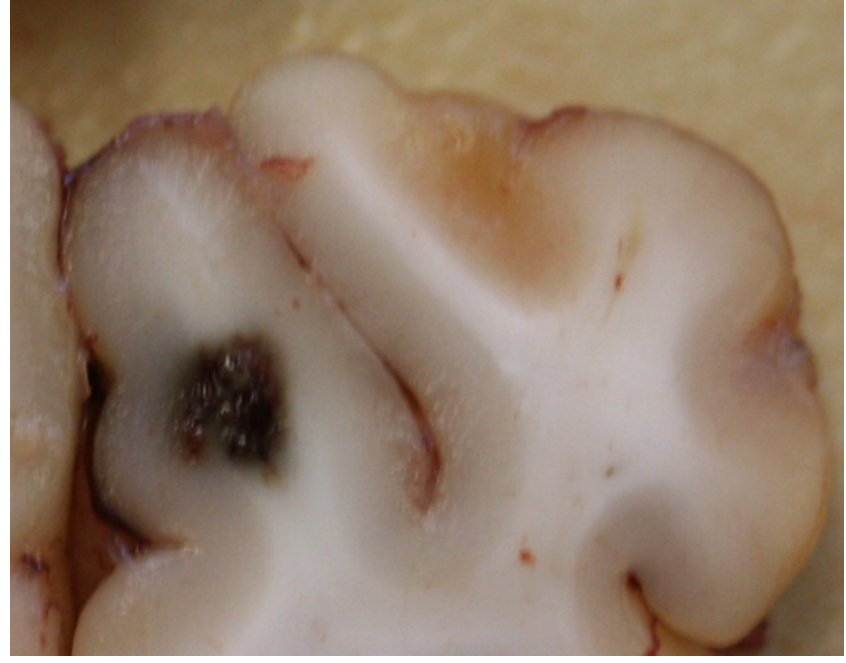

FIG. 7. Morphological section showing the large lesion from pig 4 of the subacute cohort. The lesion appears well confined and hemorrhagic in nature, with no evident damage or hemorrhage into the surrounding tissues. Figure is available in color online only.

no behavioral changes were reported and all animals remained healthy for the 72-hour recovery period.

\section{Discussion}

We have demonstrated that histotripsy can be used to generate compact, well-defined lesions in the brain with sharp boundaries between treated and untreated regions without generating damage in surrounding tissues. Using ultrasound imaging, we were able to precisely guide and monitor histotripsy treatment and accurately target individual gyri. MRI and histological evaluation showed that histotripsy damage was well confined to within only the targeted volumes and conformed well with the known focal dimensions of the histotripsy transducer $(1.5 \times 1.5 \times$ $2.5 \mathrm{~mm}$ ). Lesions were seen to be hemorrhagic in nature and were composed primarily of red blood cells, acellular debris, and/or macrophages depending on the timing. MRI and histological evaluation in the acute and subacute phases revealed no evidence of bleeding within the perilesional area.

The spatial extent of injury and boundaries of lesions did not vary as a function of applied histotripsy dose. This compares favorably with thermal (HIFU) and radiosurgery lesioning therapies, which can produce more extensive peripheral damage as the dose is increased. ${ }^{8}$ Damage within the lesions progressed from sparse to dense as the dosage was increased. This progression of damage is expected during histotripsy lesioning and is a result of the physical processes underlying bubble cloud formation during each therapy pulse. While increasing dose leads to further injury within the histotripsy focal volume, there is no additional damage to the surrounding tissues.

MRI and histological analysis indicated that hemorrhage beyond the confines of the generated lesions was minimal in the acute and subacute phases, suggesting that intracerebral hemorrhage (ICH) resulting from treatment is not an outcome inherent to the histotripsy lesioning

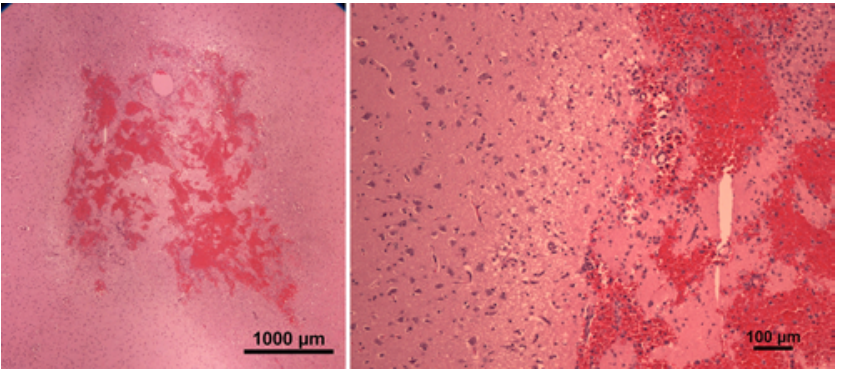

FIG. 8. H \& E-stained sections of the large lesion generated in pig 4 of the subacute study. Left: The central region is seen to show complete tissue fractionation and is filled with hemorrhage and macrophages. Right: The boundary of the lesion is sharply defined, with ischemic injuries appearing to extend out $200 \mu \mathrm{m}$ from the lesion. Adjacent tissues appear to be viable and unaffected. Figure is available in color online only.

process in the brain. While minor subarachnoid hemorrhage was observed near lesions close to boundaries, it was limited in extent and confined to the treatment area. Histopathological findings showed minimal inflammatory infiltration and an abundance of macrophages within the lesions and were generally consistent with a confined subacute infarct. Gadolinium-enhanced T1-weighted images indicated that the lesioning process did not lead to significant disruptions to the vasculature or blood-brain barrier in the perilesional area. The edema response to histotripsy lesioning in the brain was generally minimal, extending $\leq 3 \mathrm{~mm}$ from the boundaries of the larger lesions, with no edema apparent around the single focal-site lesions. These results suggest that histotripsy lesioning compares favorably with other minimally invasive to noninvasive lesioning modalities ${ }^{8}$ for brain applications over a range of lesioning metrics.

In comparison to HIFU therapies, histotripsy is able to treat large ${ }^{12}$ and superficial ${ }^{34}$ lesions in shorter durations of time. In particular, ultrasound absorption in tissues outside of the focal volume during HIFU thermal therapies leads to perilesional heating and thus has to be delivered slowly, typically on the order of $\leq 0.2 \mathrm{~cm}^{3} /$ minute..$^{25,26}$ The limitations in the speed and targeting depth of HIFU thermal therapies effectively restrict these therapies to small, central volumes within the brain that may be treated safely in clinically relevant timescales. While histotripsy is an ultrasound therapy, it uses ultrasound pulses with durations up to thousands of times shorter than those used during thermal therapies and relies on a nonthermal mechanism for lesion generation..$^{27,37,38,42,43}$ This limits the degree to which histotripsy pulses can induce heating in overlying and surrounding tissues and reduces the potential for thermal damage in the perilesional volume. This reduced heating potential allows histotripsy to be safely delivered significantly faster than HIFU thermal therapies and to target regions significantly closer to the skull surface. We have previously demonstrated in an in vitro ICH model that up to $40 \mathrm{ml}$ of clot can be liquefied within 20 minutes through intact human skulls ${ }^{12}$ (corresponding to a treatment rate of $2 \mathrm{~cm}^{3} /$ minute) while generating temperature increases in the skull bone of $\leq 4^{\circ} \mathrm{C}$, even when targeting regions as close to the skull surface as $5 \mathrm{~mm} .^{34}$

These advantages and lesioning characteristics suggest 
a number of potential uses for histotripsy in neurosurgical applications. Previous in vitro ICH studies indicate a potential for histotripsy to be used as a minimally invasive ICH therapy option that can rapidly liquefy clot volumes for aspiration ${ }^{12}$ to reduce bioburden and local mass effects. Histotripsy also has potential as a treatment option for targets in the brain such as tumors, wherein histotripsy might be used to liquefy tumor volumes, thus assisting in the resection of fibrous tumors (e.g., meningiomas). The sparse nature of damage generated by histotripsy at lower applied doses may present another potentially desirable treatment capability for multifocal tumors, such as glioblastomas. As has been demonstrated utilizing other focused ultrasound therapies, ${ }^{4}$ it may be possible to elicit an immune response against tumor targets in the brain by partially ablating them using low-dose histotripsy, thus inducing a more systemic removal of them than might have been possible otherwise.

This study was a feasibility study demonstrating the efficacy and basic safety of applying histotripsy to generate subsurface, targeted lesions in the swine brain. Among the limitations of this study were the necessity of performing a craniectomy to allow delivery of therapy to the treatment targets and the limited duration of survival following treatment to monitor for long-term effects. The craniectomy in this study was necessary due primarily to geometrical differences between the shapes of the pig skull, which is small and flat, and the propagating ultrasound pulses responsible for therapy, which are spherically converging. This geometrical mismatch results in significant ultrasound reflection at the pig skull and prevents effective transcranial delivery of treatment. As the morphology of the pig skull only permits access to the brain through one small, flat region of skull on top of the head, this constraint is specific to the swine case, and no craniectomy is expected to be necessary in humans where the skull is significantly larger and rounder. However, this study was not designed to demonstrate the in vivo feasibility of generating histotripsy lesions transcranially, just the efficacy and basic safety of histotripsy lesioning in the brain itself.

\section{Conclusions}

In this study we have utilized an in vivo porcine model to address a number of primary concerns related to delivering histotripsy in the brain and to demonstrate 1) that cerebral lesion generation may be accomplished without excess hemorrhage or other major complications associated with treatment in the acute and subacute phases after treatment, 2) that injuries to tissues surrounding the treatment target are minimal, 3) that a dose of 50 histotripsy pulses is required to fully ablate targeted brain tissue volumes, and 4) that histotripsy lesioning in the brain is versatile and accurate.

\section{Acknowledgments}

This work is supported by grants from the Focused Ultrasound Foundation, the National Institute of Biomedical Imaging and Bioengineering (NIBIB) of the National Institutes of Health under award number R01EB008998, NIH grant R01 CA134579, and the National Science Foundation (S10 RR022425).

\section{References}

1. Aronow $\mathrm{S}$ : The use of radio-frequency power in making lesions in the brain. J Neurosurg 17:431-438, 1960

2. Clement GT, White PJ, Hynynen K: Enhanced ultrasound transmission through the human skull using shear mode conversion. J Acoust Soc Am 115:1356-1364, 2004

3. Cline HE, Schenck JF, Watkins RD, Hynynen K, Jolesz FA: Magnetic resonance-guided thermal surgery. Magn Reson Med 30:98-106, 1993

4. Cohen-Inbar O, Xu Z, Sheehan JP: Focused ultrasound-aided immunomodulation in glioblastoma multiforme: a therapeutic concept. J Ther Ultrasound 4:2, 2016

5. Coluccia D, Fandino J, Schwyzer L, O'Gorman R, Remonda $\mathrm{L}$, Anon J, et al: First noninvasive thermal ablation of a brain tumor with MR-guided focused ultrasound. J Ther Ultrasound $2: 17,2014$

6. Connor CW, Hynynen K: Patterns of thermal deposition in the skull during transcranial focused ultrasound surgery. IEEE Trans Biomed Eng 51:1693-1706, 2004

7. Elias WJ, Huss D, Voss T, Loomba J, Khaled M, Zadicario E, et al: A pilot study of focused ultrasound thalamotomy for essential tremor. N Engl J Med 369:640-648, 2013

8. Elias WJ, Khaled M, Hilliard JD, Aubry JF, Frysinger RC, Sheehan JP, et al: A magnetic resonance imaging, histological, and dose modeling comparison of focused ultrasound, radiofrequency, and Gamma Knife radiosurgery lesions in swine thalamus. J Neurosurg 119:307-317, 2013

9. Fry FJ, Barger JE: Acoustical properties of the human skull. J Acoust Soc Am 63:1576-1590, 1978

10. Fry WJ, Barnard JW, Fry EJ, Krumins RF, Brennan JF: Ultrasonic lesions in the mammalian central nervous system. Science 122:517-518, 1955

11. Fry WJ, Mosberg WH Jr, Barnard JW, Fry FJ: Production of focal destructive lesions in the central nervous system with ultrasound. J Neurosurg 11:471-478, 1954

12. Gerhardson T, Sukovich JR, Pandey AS, Hall TL, Cain CA, $\mathrm{Xu} \mathrm{Z}$ : Effect of frequency and focal spacing on transcranial histotripsy clot liquefaction, using electronic focal steering. Ultrasound Med Biol 43:2302-2317, 2017

13. Hall TL, Fowlkes JB, Cain CA: A real-time measure of cavitation induced tissue disruption by ultrasound imaging backscatter reduction. IEEE Trans Ultrason Ferroelectr Freq Control 54:569-575, 2007

14. Hynynen K, Clement GT, McDannold N, Vykhodtseva N, King R, White PJ, et al: 500-element ultrasound phased array system for noninvasive focal surgery of the brain: a preliminary rabbit study with ex vivo human skulls. Magn Reson Med 52:100-107, 2004

15. Hynynen K, Jolesz FA: Demonstration of potential noninvasive ultrasound brain therapy through an intact skull. Ultrasound Med Biol 24:275-283, 1998

16. Hynynen K, Vykhodtseva NI, Chung AH, Sorrentino V, Colucci V, Jolesz FA: Thermal effects of focused ultrasound on the brain: determination with MR imaging. Radiology 204:247-253, 1997

17. Ishihara Y, Calderon A, Watanabe H, Okamoto K, Suzuki Y, Kuroda K, et al: A precise and fast temperature mapping using water proton chemical shift. Magn Reson Med 34:814823,1995

18. Jeanmonod D, Werner B, Morel A, Michels L, Zadicario E, Schiff G, et al: Transcranial magnetic resonance imaging-guided focused ultrasound: noninvasive central lateral thalamotomy for chronic neuropathic pain. Neurosurg Focus 32(1):E1, 2012

19. Kim Y, Hall T, Xu Z, Cain C: Transcranial histotripsy therapy: a feasibility study. IEEE Trans Ultrason Ferroelectr Freq Control 61:582-593, 2014

20. Lynn JG, Putnam TJ: Histology of cerebral lesions produced by focused ultrasound. Am J Pathol 20:637-649, 1944 
21. Marquet F, Pernot M, Aubry JF, Montaldo G, Marsac L, Tanter M, et al: Non-invasive transcranial ultrasound therapy based on a 3D CT scan: protocol validation and in vitro results. Phys Med Biol 54:2597-2613, 2009

22. Maxwell AD, Cain CA, Hall TL, Fowlkes JB, Xu Z: Probability of cavitation for single ultrasound pulses applied to tissues and tissue-mimicking materials. Ultrasound Med Biol 39:449-465, 2013

23. McDannold N, Clement GT, Black P, Jolesz F, Hynynen K: Transcranial magnetic resonance imaging- guided focused ultrasound surgery of brain tumors: initial findings in 3 patients. Neurosurgery 66:323-332, 2010

24. McDannold N, King RL, Hynynen K: MRI monitoring of heating produced by ultrasound absorption in the skull: in vivo study in pigs. Magn Reson Med 51:1061-1065, 2004

25. Monteith SJ, Harnof S, Medel R, Popp B, Wintermark M, Lopes MB, et al: Minimally invasive treatment of intracerebral hemorrhage with magnetic resonance-guided focused ultrasound. J Neurosurg 118:1035-1045, 2013

26. Monteith SJ, Kassell NF, Goren O, Harnof S: Transcranial MR-guided focused ultrasound sonothrombolysis in the treatment of intracerebral hemorrhage. Neurosurg Focus 34(5):E14, 2013

27. Parsons JE, Cain CA, Abrams GD, Fowlkes JB: Pulsed cavitational ultrasound therapy for controlled tissue homogenization. Ultrasound Med Biol 32:115-129, 2006

28. Pernot M, Aubry JF, Tanter M, Thomas JL, Fink M: High power transcranial beam steering for ultrasonic brain therapy. Phys Med Biol 48:2577-2589, 2003

29. Pichardo S, Hynynen K: Treatment of near-skull brain tissue with a focused device using shear-mode conversion: a numerical study. Phys Med Biol 52:7313-7332, 2007

30. Pichardo S, Sin VW, Hynynen K: Multi-frequency characterization of the speed of sound and attenuation coefficient for longitudinal transmission of freshly excised human skulls. Phys Med Biol 56:219-250, 2011

31. Pinton G, Aubry JF, Bossy E, Muller M, Pernot M, Tanter M: Attenuation, scattering, and absorption of ultrasound in the skull bone. Med Phys 39:299-307, 2012

32. Pinton G, Aubry JF, Fink M, Tanter M: Effects of nonlinear ultrasound propagation on high intensity brain therapy. Med Phys 38:1207-1216, 2011

33. Sukovich J, Xu Z, Kim Y, Cao H, Nguyen TS, Pandey A, et al: Targeted lesion generation through the skull without aberration correction using histotripsy. IEEE Trans Ultrason Ferroelectr Freq Control 63:671-682, 2016

34. Sukovich JR, Xu Z, Hall TL, Allen SP, Cain CA: Efficacy and treatment envelope of transcranial histotripsy therapy without using aberration correction. J Ther Ultrasound 4 (Suppl 1):A127, 2016 (Abstract)

35. Sweet WH, Mark VH, Hamlin H: Radiofrequency lesions in the central nervous system of man and cat: including case reports of eight bulbar pain-tract interruptions. J Neurosurg 17:213-225, 1960

36. Vlaisavljevich E, Kim Y, Allen S, Owens G, Pelletier S, Cain $\mathrm{C}$, et al: Image-guided non-invasive ultrasound liver ablation using histotripsy: feasibility study in an in vivo porcine model. Ultrasound Med Biol 39:1398-1409, 2013

37. Vlaisavljevich E, Kim Y, Owens G, Roberts W, Cain C, Xu $\mathrm{Z}$ : Effects of tissue mechanical properties on susceptibility to histotripsy-induced tissue damage. Phys Med Biol 59:253270,2014

38. Vlaisavljevich E, Maxwell A, Warnez M, Johnsen E, Cain $\mathrm{CA}, \mathrm{Xu} \mathrm{Z}$ : Histotripsy-induced cavitation cloud initiation thresholds in tissues of different mechanical properties.
IEEE Trans Ultrason Ferroelectr Freq Control 61:341352,2014

39. Wang TY, Xu Z, Winterroth F, Hall TL, Fowlkes JB, Rothman ED, et al: Quantitative ultrasound backscatter for pulsed cavitational ultrasound therapy-histotripsy. IEEE Trans Ultrason Ferroelectr Freq Control 56:995-1005, 2009

40. Wheat JC, Hall TL, Hempel CR, Cain CA, Xu Z, Roberts WW: Prostate histotripsy in an anticoagulated model. Urology 75:207-211, 2010

41. White PJ, Clement GT, Hynynen K: Longitudinal and shear mode ultrasound propagation in human skull bone. Ultrasound Med Biol 32:1085-1096, 2006

42. Xu Z, Fowlkes JB, Rothman ED, Levin AM, Cain CA: Controlled ultrasound tissue erosion: the role of dynamic interaction between insonation and microbubble activity. J Acoust Soc Am 117:424-435, 2005

43. Xu Z, Ludomirsky A, Eun LY, Hall TL, Tran BC, Fowlkes JB, et al: Controlled ultrasound tissue erosion. IEEE Trans Ultrason Ferroelectr Freq Control 51:726-736, 2004

44. Young GF, Lele PP: Focal lesions in the brain of growing rabbits produced by focused ultrasound. Exp Neurol 9:502511,1964

45. Zhang X, Owens GE, Cain CA, Gurm HS, Macoskey J, Xu Z: Histotripsy thrombolysis on retracted clots. Ultrasound Med Biol 42:1903-1918, 2016

46. Zhang X, Owens GE, Gurm HS, Ding Y, Cain CA, Xu Z: Noninvasive thrombolysis using histotripsy beyond the intrinsic threshold (microtripsy). IEEE Trans Ultrason Ferroelectr Freq Control 62:1342-1355, 2015

\section{Disclosures}

Drs. Cain, Hall, and Zhen Xu report an ownership interest in HistoSonics, Inc. Dr. Cannata reports an employee relationship with and ownership interest in HistoSonics, Inc. Dr. Bertolina reports patent holder and employee relationships with and direct stock ownership in HistoSonics, Inc. Dr. Kassell reports an ownership interest in Insightec.

\section{Author Contributions}

Conception and design: Sukovich, Cain, Hall, Zhen Xu. Acquisition of data: Sukovich, Pandey, Allen, Snell, Zhiyuan Xu. Analysis and interpretation of data: Sukovich, Pandey, Chaudhary, Camelo-Piragua, Allen, Snell. Drafting the article: Sukovich, Pandey, Zhen Xu. Critically revising the article: Sukovich, Cain, Pandey, Camelo-Piragua, Allen, Hall, Snell, Zhen Xu. Reviewed submitted version of manuscript: Sukovich, Pandey, Snell. Approved the final version of the manuscript on behalf of all authors: Sukovich. Administrative/technical/material support: Cain, Hall, Cannata, Teofilovic, Bertolina, Kassell, Zhen Xu. Study supervision: Cain, Hall, Kassell, Zhen Xu. Built the histotripsy transducer: Hall. Performed the craniectomy surgery on the pigs during the experiments at UVA: Zhiyuan Xu.

\section{Supplemental Information \\ Previous Presentations}

Portions of this work were presented at the 5th International Symposium of Focused Ultrasound, Bethesda, Maryland, August 30, 2016.

\section{Correspondence}

Jonathan R. Sukovich: University of Michigan, Ann Arbor, MI. jsukes@umich.edu. 